\title{
Evaluation approaches of fish swimming performance
}

\author{
Fukun Gui, Ping Wang*, Changwen Wu \\ Zhejiang Key Lab of Marine Aquaculture Facilities and Engineering Technology, Zhejiang Ocean University, Zhoushan, China; \\ *Corresponding Author: wp77319@163.com
}

Received 10 November 2013; revised 13 January 2014; accepted 28 January 2014

Copyright (C) 2014 Fukun Gui et al. This is an open access article distributed under the Creative Commons Attribution License, which permits unrestricted use, distribution, and reproduction in any medium, provided the original work is properly cited. In accordance of the Creative Commons Attribution License all Copyrights (C) 2014 are reserved for SCIRP and the owner of the intellectual property Fukun Gui et al. All Copyright (C) 2014 are guarded by law and by SCIRP as a guardian.

\section{ABSTRACT}

Swimming speeds are the most important index for the evaluation of the fish swimming performance. The terminologies and classifications of the fish swimming performance were summarized in this paper. Taking into consideration of the widely used evaluation approaches of the fish swimming performance by different researchers, a recommended classification methodology of the fish swimming performance was proposed by the authors. And a new concept of the swimming speed, the Maximum Domed Swimming Speed (DSS), was introduced into this new classification framework together with a discussion on its calculation method and the practical significance. According to the classification system, the fish swimming speeds are classified into five categories: Optimum Swimming Speed, Maximum Sustained Swimming Speed, Critical Swimming Speed, Maximum Domed Swimming Speed, and Burst Swimming Speed. Other concepts of swimming speeds are generally merged into the above five categories, respectively. Furthermore, possible relevancies among the Maximum Sustained Swimming Speed (MSS), the Critical Swimming Speed (CSS), and the Maximum Domed Swimming Speed (DSS) were discussed. It was concluded that these three swimming speeds, in a sense, can be regarded as the equivalent indices for the evaluation of fish swimming performance.

\section{KEYWORDS}

Swimming performance; Optimum Swimming Speed; Maximum Sustained Swimming Speed; Critical Swimming Speed; Maximum Domed

\section{Swimming Speed; Burst Swimming Speed}

\section{INTRODUCTION}

Swimming performance is considered as a critical character determining the survival of many fishes in the natural circumstance [1]. For many fishes, swimming is the main way to avoid the attack from their predators, obtain food, find a mate, and so on [2,3]. Swimming speed and swimming time are the two mainly used indices for evaluating swimming capability of fishes. Plaut [4] and Hammer [5] summarized the evaluation approaches of the fish swimming performance used by different researchers, and classified them into three major categories: Cruising Swimming Speed (or Sustained Swimming Speed), Prolonged Swimming Speed and Burst Swimming Speed. This classification methodology is based on the swimming time durations that fishes were swum. The Cruising Swimming Speed (or Sustained Swimming Speed) defines those speeds that can be maintained by a fish for more than 200 min without exhaustion [6]. The prolonged swimming is of shorter duration of time from 20s to 200 min than cruising [7], and ends in fatigue of fish. Burst swimming can only be maintained for brief periods of less than approximately $20 \mathrm{~s}$ [8,9].

However, while doing so, only a few authors adhere to the experimental protocols originally suggested. Instead, they applied a great variety of durations for testing the swimming speeds of fishes. Take the sustained swimming speed as an example, the swimming time durations of $4 \mathrm{~h} \mathrm{[8],} 6 \mathrm{~h} \mathrm{[10],} 12 \mathrm{~h}, 24 \mathrm{~h}$ and $48 \mathrm{~h}$ [11,12] were used frequently. The Critical Swimming Speed has been widely accepted as an efficient measurement of prolonging the swimming test. However, the length of tests will also exceed largely the time limitation of the prolonged swimming as defined above when small velocity in- 
creasing steps and long-time intervals were applied. Although the classification has been accepted by some other authors $[13,14]$, no significant ecological or physiological relevance has been proposed to validate the classification by defining the cruising swimming speed as the speed that can be maintained by a fish for more than 200 min. Moreover, by doing so, the confusion and misunderstanding may occur when comparing the swimming performance among different fishes.

The main purpose of this paper is to propose a new classification of the swimming speeds according to the swimming status of fishes without strict limitation to their swimming time durations. And a new concept of swimming speed, the Maximum Domed Swimming Speed, was introduced into this new classification framework together with a discussion on its calculation and the practical significance.

\section{FISH SWIMMING PERFORMANCE}

Briefly, we classified the swimming status into continuous swimming and transient swimming, as shown in Figure 1. Transient swimming is performed anaerobically and propelled by the white musculature. According to the results from many authors, a maximal endurance of 15 - $20 \mathrm{~s}$ in transient swimming status was generally found and accepted [7,9,15]. Continuous swimming is fuelled aerobically and can be sustained by a fish for a relative long time, and locomotion is mainly propelled by the red musculature. However, the white musculature will also be used when a fish is exposed to strong currents or variable currents $[16,17]$. Swimming performance can be classified further into sublevels, as shown in Figure 1, which will be discussed below in details.

\section{STEADY SWIMMING PERFORMANCE}

In steady swimming status, fishes are exposed to a certain velocity which is not altered during the test. Generally, it is also called cruising swimming or sustained swimming [18] and accordingly, the speed is called cruising swimming speed or sustained swimming speed. Cruising speed is originally introduced from aviation domain, which defines the speed that an aerocraft can keep flying with minimal energy consumption. However, it is difficult to define quantitatively when it is used for evaluating the swimming performance of a fish because a series of factors should be considered including fatigue, growing, health, and so on. Normally, it is regarded that fish will not end in fatigue in cruising swimming. But theoretically, a fish will fatigue at any speed, given the time duration long enough without feeding. Thus, when discussing cruising swimming speeds, the research purposes or the swimming time must be determined in advance.

According to the discussion above, we classified the following two concepts: the Optimum Cruising Swimming Speed and the Maximum Cruising (or Sustained) Swimming Speed into the steady swimming performance. A research purpose such as foraging, growth rate, migrating distance, and so on, must be determined when the former concept is used, and the period of swimming time is needed for the latter. For example, Ware [19] derived theoretically that, with regard to bioenergetics, fish has an optimum foraging speed. Taking Oncorhynchus nerka as an example, he found it is proportional to 0.4 power of the body length. This means, from the point of view of bioenergetics, the cruising speed of Oncorhynchus nerka is not the lower the better. And this was also verified by

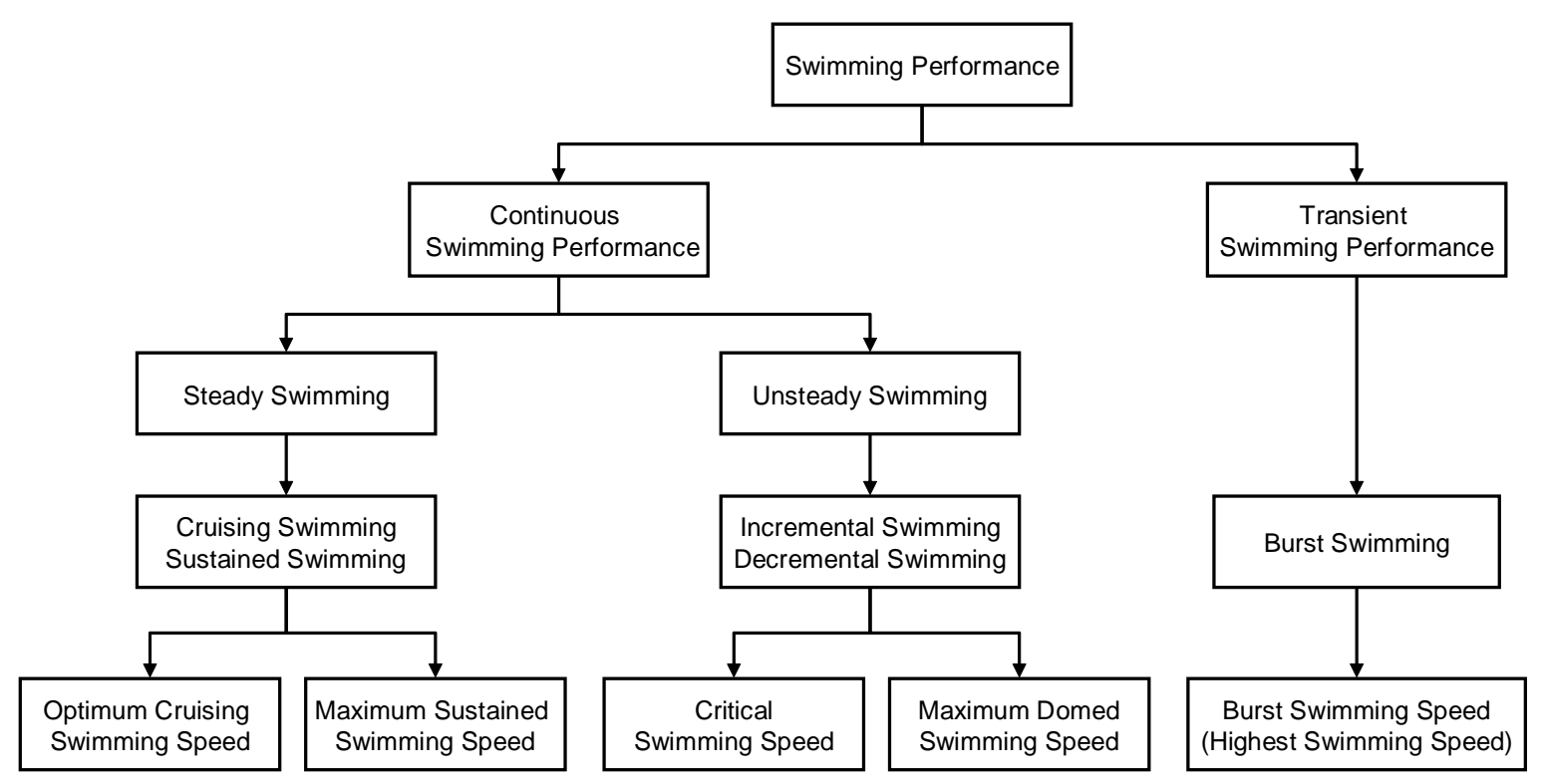

Figure 1. Classification of swimming performance of fishes. 
Hammer's [20] experiments on the growth rate of Merlangius merlangus, which showed a much higher growth rate at the swimming speed of $0.28 \mathrm{BL} / \mathrm{s}$ than that at the swimming speed of $0.14 \mathrm{BL} / \mathrm{s}$. Trump and Leggett [21] also calculated the optimum cruising speeds for fish migrating in currents. The concepts proposed or derived from those researches, such as the Optimum Foraging Swimming Speed, the Optimum Migrating Swimming Speed and the Optimum Growing Swimming Speed can fall under the Optimum Cruising Swimming Speed. However, it is still difficult to draw general conclusions on the definitions and researching approaches of different cruising swimming speeds due to the very divergent methods that have been used [5].

The Maximum Cruising (or Sustained) Swimming Speed refers to the maximal steady speed that a fish can withstand for a certain length of swimming time and the fish will end in fatigue. Customarily, the concept, Maximum Sustained Swimming Speed (MSS) was used more often by many authors than the Maximum Cruising Swimming Speed because the word "cruising" is normally regarded as the sign of swimming status of a fish without fatigue. Apparently, the MSS depends solely on the swimming time with other conditions unchanged. A period of swimming time less than $20 \mathrm{~s}$ is regarded as the range of transient swimming status, and thus $20 \mathrm{~s}$ is normally accepted as the lower limit for a fish to maintain sustained swimming status [7,9]. Theoretically, there is no limitation to the maximum swimming time. But the longer the swimming time is given, the lower the MSS will be. It is difficult, if not impossible, to measure the MSS directly. However, it is rather easy to record the swimming times of the fishes at different steady speeds in laboratory experiments. Therefore, it is definitely possible to set up a regression relationship between the swimming speed and the swimming time, and the MSS can be obtained by interpolating in the regression curve according to the designated swimming time that a fish will be forced to swim [22]. There are several standards for measuring the swimming time durations in the laboratory tests. Brett [6] and Fisher and Wilson [12] used a certain ratio of fishes fatigued, i.e. $50 \%$ and $10 \%$ respectively, as the ending standard of each test. Fisher and Bellwood [11] used an average time standard, which means that each individual fish ended in fatigue and its swimming time duration was recorded separately. Generally, the former standard will be used when a large quantity of fishes are going to be tested. While the latter one is basically applied to the cases with small samples.

\section{UNSTEADY SWIMMING PERFORMANCE}

Fish is exposed to varying speeds in unsteady swimming status which includes incremental swimming and decremental swimming. To date, almost all the tests of unsteady swimming performance were carried out in the incremental swimming status. The spontaneous swimming is, needless to say, a status of unsteady swimming but not an index for evaluating a fish's swimming performance. A fish exposed to waves may also be regarded as a case being in unsteady swimming status. However, this is a very complicated topic and to date there is no relative result been published. Considering about its specialty, we suggest carrying out the research works in this field separately and it will not be discussed in this paper.

To date, the Critical Swimming Speed (CSS) is the most important index to evaluate the unsteady swimming performance [23-27]. It is measured using incremental swimming approach. The current velocities are not increased gradually, but rather in steps, each speed being maintained for a certain period of time until exhaustion occurs [5]. However, controversy has existed for a long time with respect to the influences of time interval and velocity increment on the CSS. Some authors demonstrated that there are no significant differences in the CSS with different protocols $[27,28]$, while some authors found that the influence on the CSS is quite notable [29-31]. Also some authors argued that the influence on the CSS can be neglected within a certain range of time interval and velocity increment $[7,22]$. On the basis of the presented findings, it is recommended to use the time intervals ranging from $20 \mathrm{~min}$ to $60 \mathrm{~min}$, and the velocity increments between one-quarter and one-ninth of the CSS which is measured through pre-experiments $[5,7]$.

The CSS has been favored largely because it takes a shorter time to conduct and uses smaller batches of fish for a statically meaningful value $[5,27,32]$. However, the CSS protocol is sometimes still time consuming and is not without limitations. A long period of testing time is required when large time intervals and small velocity increments are used in the tests. Therefore, some other approaches were proposed to cut down the time durations in the tests. Jain et al. [24] tested the swimming performance of rainbow trout (Oncorhynchus mykiss) by using the Ramp Velocity Test protocol. This protocol requires a rapid increase of velocities to $75 \%$ of the CSS within 5 - 6 min at the first stage. Subsequently, the time intervals are fixed on 30 min till the fish fatigues. Farrel [27] developed the Constant Acceleration Velocity Test protocol, in which a very short time interval of $1 \mathrm{~min}$ duration is used. In fact, this approach can be regarded as a special case of the CSS with extreme short time interval. However, it will, to large extent, overestimate the value of the CSS though much time is saved.

It is generally assumed that the CSS is the speed at which the maximum oxygen uptake occurs [26], and is regarded as a repeatable testing approach. However, the physiological and ecological significance of the CSS is 
still open to question [5,33]. Although some authors tried to find out its physiological and ecological relevancies through some statistical tools, many conclusions rest only on hypothesizes or speculations [4]. And the conditions, used in the tests, are far from representing most natural environment experienced by fishes [4]. However, if we don't care too much about the test time duration, the CSS can, in a sense, represent the swimming performance of a fish exposed to a natural tidal current variation. If this is the situation, we would like to say the CSS has its real meaning. In the real sea, the water current follows a certain tidal principle. Take the ideal semidiurnal tide as an example, the tidal current increases from zero to the maximum velocity within approximately $3 \mathrm{~h}$ and drops down to zero again in the next $3 \mathrm{~h}$. This process repeats endlessly. The first stage is an incremental process, which is more or less similar to that of the CSS protocol. The second stage is a decremental process. To date, there is no published result of fish swimming performance tested in this condition. The fishes constrained in an off-shore sea cage are exposed to the tidal current all the day, and it is very important for the fishes to survive in the attack form the tidal current. This means that the fishes have to finish the whole process of the half period (approximate $6 \mathrm{~h} 12 \mathrm{~min}$ ) without fatigue.

The maximal tidal current velocity is the key factor determining the final condition of a fish exposed to tidal current. If a fish can finish the whole process of a half tidal period (approximate $6 \mathrm{~h} 12 \mathrm{~min}$ for semi-diurnal tide) and happens to be fatigued in the end, the maximal tidal current velocity is called the Maximum Domed Swimming Speed (DSS). The DSS is very difficult, if not impossible, to measure either in the laboratory or in the natural environment. The authors proposed a semi-empirical \& theoretical method for calculating the DSS, which is based on the tidal current theory and the empirical relationship between the maximum sustained swimming speed and the maximum sustained swimming time. This will be discussed more in the following.

\section{BURST SWIMMING PERFORMANCE}

In many fishes, the Burst Swimming Speed (BSS), anaerobic speed, is more useful to avoid predator attack, gain food, escape from trawls than sustained swimming speed [4,34], and is critical to their survival. It is generally accepted that burst swimming is performed anaerobically and it can only be maintained for a very short periods of less than 15 - $20 \mathrm{~s}$ [7,9]. According to Webb [35], bursts may also include steady or unsteady swimming status. For many species, the BSS can be estimated with the 10BL/s-rule [20,36,37]. However, the BSS depends very much on the duration of the performed burst, which declines exponentially with time and increases with size in absolute units $(\mathrm{cm} / \mathrm{s})$, but decreases in relative units (BL/s) [5].

\section{MAXIMUM DOMED SWIMMING SPEED}

The Maximum Domed Swimming Speed (DSS) refers to the maximal tidal current velocity that a fish can finish the time duration of a half tidal period and happens to be fatigued in the end. During this process, the fish will be exposed to the currents which will increase from zero to the DSS and drop down to zero again according to the tidal principle. The DSS has practical meanings as it can be used for selection of suitable fish farming sites.

As mentioned above, the DSS is difficult to be obtained through directly laboratory measurements. A mediate method is needed. Again, we take the ideal semidiurnal tidal current as an example. The values and directions of tidal current will vary with time, which are controlled by the half-day period and the half-month period, and can be calculated using the following formula [38].

$$
V_{t}=V_{m n} \cdot \sin \left(\frac{2 \pi}{T_{C}} t\right)+K \cdot V_{m n} \cdot \sin \left(\frac{2 \pi}{T_{m n}} t\right) \cdot \sin \left(\frac{2 \pi}{T_{C}} t\right)
$$

where, $V_{t}$ is the tidal current velocity at time $t$. The first term in the right part is the velocity variation in the half-day period and is followed by that in the half-month period. $V_{m n}$ is the average velocity amplitude which is written as:

$$
V_{m n}=\frac{V_{m}+V_{n}}{2}
$$

where, $V_{m}$ and $V_{n}$ refer to the maximum tidal current velocities in spring tide and neap tide, respectively. $K$ is the coefficient of velocity fluctuation and is calculated by:

$$
K=\frac{V_{m}-V_{n}}{V_{m}+V_{n}}
$$

$T_{C}$ in Formula (1) is the half-day period, which equals to $12.4 \mathrm{~h} ; T_{m n}$ is the half-month period, which equals to $14.75 \mathrm{~d}$. For evaluation the swimming performance of a fish, the half-day period $\left(T_{C}=12.4 \mathrm{~h}\right)$ is long enough. Thus, only the first term in the right part of Formula (1) is used and the average velocity amplitude $V_{m n}$ is substituted by the maximal tidal current velocity $V_{m}$, i.e.

$$
V_{t}=V_{m} \cdot \sin \left(\frac{2 \pi}{T_{C}} t\right)
$$

Within the half-day period $t \in\left(0, T_{C}\right)$, the current velocity will reach the same maximum values twice, but run in opposite directions. Fortunately, the current direction has no influence to the fish swimming performance. Therefore, it is can be discussed within $t \in\left(0, T_{C} / 2\right)$, 
namely $t \in(0,6.2 h)$, in which the velocity will increase from zero to its maximal value $V_{m}$ and drop down to zero again. Here, $V_{m}$ is the very speed of the DSS.

In order to obtain $V_{m}$, the DSS, we have to correlate Formula (4) to fish swimming performance. According to some authors [11,12], there is a kind of exponential relationship between the maximum sustained swimming time and the MSS, which is written as:

$$
T=a V^{b}
$$

where, $T$ means the maximum sustained swimming time when a fish is forced to swim at velocity $V$ (the MSS); $a$ and $b$ are the coefficients which can be obtained through experimental measurements and statistical analysis. By substituting (4) into (5), we have:

$$
T=a \cdot V_{m}{ }^{b} \cdot \sin ^{b}\left(\frac{2 \pi}{T_{C}} t\right)
$$

where, $T$ means the maximum sustained swimming time of a fish exposed to the tidal current velocity at time $t$. The Critical Swimming Speed $\left(U_{c s s}\right)$ is calculated using the following formula as originally given by Brett [23]:

$$
U_{c s s}=U_{p}+\left[\left(t_{f} / t_{i}\right) \times U_{i}\right]
$$

where, $U_{p}$ is the penultimate velocity at which the fish swam before fatigue; $U_{i}$ is the velocity increment $(\mathrm{cm} / \mathrm{s}) ; t_{f}$ is the elapsed time in the last velocity stage; $t_{i}$ is the time interval (normally form $20 \mathrm{~min}$ to $60 \mathrm{~min}$ [5]). From the second term in the right part of formula (7), we can find that the stamina of a fish is assumed to be distributed uniformly within the swimming time interval $t_{i}$. Here, we also follow this assumption: the stamina of a fish is distributed uniformly during the whole stage of steady swimming. Thus, if we divide the swimming duration $T_{C} / 2$ into many small time fragments $d t$, the ratio of the stamina consumption within $d t$ will be $d t / T$ (not $d t / T_{C}$ ). Where, $T$ is the maximum sustained swimming time at velocity $V_{t}$. By integrating the stamina consumption within the whole testing period $T_{C} / 2$, the value will equal to 1 if the fish happens to be fatigued in the end. Namely, we have:

$$
\int_{0}^{T_{C} / 2} \frac{1}{T} \mathrm{~d} t=1 \Rightarrow \int_{0}^{T_{C} / 2} \frac{1}{a V_{m}^{b} \sin ^{b}\left(\frac{2 \pi}{T_{C}} t\right)} \mathrm{d} t=1
$$

The $V_{m}$, i.e. the DSS, can be calculated by Formula (8) through numerical integrating. However, the DSS depends on the coefficients $a$ and $b$ which are obtained through experimental tests. This means that the DSS is relevant to the MSS. They may, in a sense, be regarded as the equivalent index for evaluating the swimming performance of fishes.

\section{DISCUSSION}

\subsection{Classification Methodology of Fish Swimming Speed/Performance}

Swimming speeds including Sustained Swimming Speed, Critical Swimming Speed and Burst Swimming Speed are the most commonly used indices for evaluating fish swimming performance in the past several decades. Researching works on swimming speeds including their definitions, measuring approaches, affecting factors and the interrelationships have been carried out extensively. Hammer [5] and Plaut [4] gave a very comprehensive summarization and discussion, and classified the swimming speeds into three categories according to the swimming time durations. However, much wider time range had been used by different authors in rather random ways, which makes the classification be inconsistent and of little sense.

Taking into consideration of the widely used evaluation approaches of fish swimming performance by different researchers, a new classification methodology was proposed by the authors of this paper. And a new concept of swimming speed, the DSS, was introduced into this new classification framework together with a discussion on its calculation method and practical significance. According to the new classification system, fish swimming speeds are classified into five major categories: Optimum Cruising Swimming Speed (OSS), Maximum Sustained Swimming Speed (MSS), Critical Swimming Speed (CSS), Maximum Domed Swimming Speed (DSS), and Burst Swimming Speed (BSS), as shown in Figure 1. Other concepts of swimming speeds are generally merged into above five categories respectively. The Optimum Foraging Swimming Speed, the Optimum Migrating Swimming Speed and the Optimum Growing Swimming Speed et al. fall down to the Optimum Cruising Swimming Speed. However, the Cruising Swimming Speed, the Sustained Swimming Speed and the Spontaneous Swimming Speed are not indices for evaluating the swimming performance of a fish. Generally, they describe some kinds of intermediate swimming status. The Prolonged Swimming Speed and the Endurance Swimming Speed are used as synonyms [5]. Both are restricted to swimming time duration from $20 \mathrm{~s}$ to $200 \mathrm{~min}[4,5]$ and have no exact meanings. Thus, it is suggested to avoid using them as the evaluation indices of fish swimming performance.

\subsection{Influence of Testing Protocols on Fish Swimming Performance}

It has always been a question about the influence of testing protocols on fish swimming performance. With regarding to the CSS, the influence of time intervals and velocity increments on its result has caught many atten- 
tions [22,27]. However, it still cannot draw a general conclusion on how the testing protocols will affect the final values of the CSS. Hammer [5] suggested using the time intervals between $20 \mathrm{~min}$ and $60 \mathrm{~min}$, and the velocity increments from one-quarter to one-ninth of the CSS as a general testing protocol. However, the time interval suggested could be a limitation for fish larvae. A much shorter time interval of 2 min was used by Fisher $[22,39]$ during the CSS measurements of several species of reef fish larvae. In addition, there are still many uncertainties due to other influence factors, which make the testing results from different authors incomparable.

Despite the defectives of the CSS, it has been used extensively as an important index of fish swimming performance because of the smaller batches and less experimental time to run the tests [5,27]. By contrast, more batches and longer time are needed to run the MSS tests, and the numerical regression and interpolation processes are needed to obtain the final values of the MSS. However, the MSS within any time durations and the maximum sustained swimming time at any speeds can be figured out once the regression relationship between them has been set up. In addition, it is a good way for investigating the biochemical changes, such as fat, glycogen and lactate, in muscles and livers of fishes [40].

Before carrying out the tests of the MSS, the fishes should be introduced into a flume tank and be acclimatized to the temperature, light, original current, and so on. After a period of acclimation, the current is increased steadily [8], in small steps [41], semi-abruptly [42], or abruptly [43], until the test speed is reached. However, the influence of the velocity increasing rate and acclimation current velocity before test on the result of the MSS is not defined or is not reported [5,8]. The authors of this paper have carried out a preliminary investigation of the influences of the acclimation current velocity and the acceleration rate on the MSS of Sciaenops ocellatus (average body length $36.3 \mathrm{~cm}$ ) and Sparus macrocephalus (average body length $23.6 \mathrm{~cm}$ ). Results show that the acclimation velocities under $1.5 \mathrm{BL} / \mathrm{s}$ (the maximum current batch in the tests) have no significant influence on the MSS of both fishes. The same results have been achieved in the acceleration tests, where the accelerating time from the original acclimation velocities to the test speeds are between $1 \mathrm{~min}$ and $8 \mathrm{~min}$. If these preliminary conclusions are verified by wider tests with different fish species and sizes, it will be very convenient to measure the MSS. If this is the situation, the results measured with different acclimation velocities and acceleration rates, within a certain range, can be compared directly.

\subsection{Interrelationships amongst MSS, CSS and DSS}

Both the DSS and the CSS belong to the unsteady swimming speeds. The DSS is tested according to the tidal current variation law, while the CSS testing uses an artful protocol in a rather random way. However, they are similar in the process as one DSS test covers double CSS tests without considering about the influence of the change of velocity orientations (increase or decrease) on the results. Therefore, a kind of relationship may be found through substantial tests on both speeds. Once the relationship between them has been set up, the swimming speeds can be converted each other. The most important is the CSS will possess a practical meaning. But before doing so, the influences of the velocity orientation, velocity increment, time duration, time interval, and so on, on the final values of swimming speeds, should be investigated in advance.

In the other hand, the DSS can be figured out using Formula (8) which is derived based on the mathematical regression model of the MSS as Formula (5). This means a certain relationship between the DSS and the MSS definitely exists. However, an assumption was introduced from the CSS Formula (7) into DSS Formula (8): the stamina of a fish is distributed uniformly during the whole period of steady swimming. This assumption is never proposed but has been used naturally in the CSS calculation. Anyway, more verification on its validity is needed. The authors of this paper made some preliminary tests on the DSS of six Sciaenops ocellatus (caught from a fishing cage with average body length of $36.3 \mathrm{~cm}$ ). During the tests, the DSS was $76 \mathrm{~cm} / \mathrm{s}$ calculated by formula (8). The velocity increased from zero to 76 $\mathrm{cm} / \mathrm{s}$ and then decreased to zero again according to the semidiurnal law. Although all the six fishes passed the maximum velocity, they stopped swimming at different time on the decreasing stage. The results are rather discrete. A possible reason is that the fishes had been cultured in a laboratory tank for 11 days and had been used for several other experimental tests. More tests and new scenarios are probably needed for further verifications.

As discussed above, we may draw a general conclusion that these three swimming speeds are, to some extent, the same indices for evaluating fish swimming performance. Amongst these three speeds, the DSS has a very clear practical meaning. It should be chosen as an important index for the selection of fish farming sites. Furthermore, it is recommended to use the MSS for evaluating the swimming performance of freshwater fishes, while the CSS and DSS may have little meaning because the river currents are generally steady.

\section{ACKNOWLEDGEMENTS}

This work was financially supported by the National Science and Technology Support Program No.51109187, the Zhejiang Provincial Climbing Program No. pd2013217, and the project form Zhoushan 
Science and Technology Bureau, No.2013C41002.

\section{REFERENCES}

[1] Stobutzki, I.C. and Bellwood, D.R. (1994) An analysis of the sustained swimming abilities of pre-settlement and post-settlement coral reef fishes. Journal of Experimental Marine Biology and Ecology, 175, 275-286.

[2] Watkins, T.B. (1996) Predator-mediated selection on burst swimming performance in tadpoles of the Pacific tree frog, Pseudacris regilla. Physiological Zoology, 69, 154-167. http://dx.doi.org/10.1016/0022-0981(94)90031-0

[3] Drucker, E.G. (1996) The use of gait transition speed in comparative studies of fish locomotion. American Zoologist, 36, 555-566.

[4] Plaut, I. (2001) Critical swimming speed: Its ecological relevance. Comparative Biochemistry and Physiology Part A, 131, 41-50. http://dx.doi.org/10.1016/S1095-6433(01)00462-7

[5] Hammer, C. (1995) Fatigue and exercise tests with fish. Comparative Biochemistry and Physiology Part A, 112, 1-20.

[6] Brett, J.R. (1967) Swimming performance of sockeye salmon (Oncorhynchus nerka) in relation to fatigue time and temperature. Journal of the Fisheries Research Board of Canada, 24, 1731-1741. http://dx.doi.org/10.1139/f67-142

[7] Beamish, F.W.H. (1978) Swimming capacity. Fish physiology. Academic Press, New York.

[8] Beamish, F.W.H. (1966) Swimming endurance of some Northwest Atlantic fishes. Journal of the Fisheries Research Board of Canada, 23, 341-347. http://dx.doi.org/10.1139/f66-028

[9] Blaxter, J.H.S. (1969) Swimming speeds of fish. FAO Conference on Fish Behavior in Relation to Fishing Techniques and Tactics, Bergen, 19-27 October 1967.

[10] Brett, J.R., Hollands, M. and Alderdice, D.R. (1958) The effect of temperature on the cruising speed of young sockeye and coho salmon. Journal of the Fisheries Research Board of Canada, 15, 587-605. http://dx.doi.org/10.1139/f58-031

[11] Fisher, R. and Bellwood, D.R. (2002) The influence of swimming speed on sustained swimming performance of late stage reef fish larvae. Marine Biology, 140, 801-807. http://dx.doi.org/10.1007/s00227-001-0758-5

[12] Fisher, R. and Wilson S.K. (2004) Maximum sustainable swimming speeds of late stage larvae of nine species of reef fishes. Journal of Experimental Marine Biology and Ecology, 312, 171-186.

http://dx.doi.org/10.1016/j.jembe.2004.06.009

[13] He, P. and Wardle, C.S. (1988) Endurance at intermediate swimming speeds of Atlantic mackerel (Scomber scombrus L.), herring (Clupea harengus) and saithe (Pollachius virens L.). Journal of Fish Biology, 33, 255-266. http://dx.doi.org/10.1111/j.1095-8649.1988.tb05468.x

[14] He, P. (1991) Swimming endurance of the Atlantic cod
(Gadua morhua L.) at low temperatures. Fisheries Research, 12, 65-73.

http://dx.doi.org/10.1016/0165-7836(91)90050-P

[15] Bailey, D.M., Bagley, P.M., Jamieson, A.J., Collins, M.A. and Priede, I.G. (2003) In situ investigation of burst swimming and muscle performance in the deep-sea fish Antimora rostrata. Journal of Experimental Marine Biology and Ecology, 285, 295-311.

[16] Webb, P.W. (197l) The swimming energetics of trout. II. Oxygen consumption and swimming energetics. The Journal of Experimental Biology Part A, 55, 521-540.

[17] Webb, P.W. (1971) The swimming energetics of trout. I. Thrust and power output at cruising speeds. The Journal of Experimental Biology Part B, 55, 489-520.

[18] Farrell, A.P. and Steffensen, J.F. (1987) Coronary ligation reduces maximum sustained swimming speed in Chinook salmon, Oncorhynchus tshawytscha. Comparative Biochemistry and Physiology Part A, 87, 35-37.

[19] Ware, D.M. (1978) Bioenergetics of pelagic fish: Theoretical change in swimming speed and ration with body size. Journal of the Fisheries Research Board of Canada, 35, 220-228. http://dx.doi.org/10.1139/f78-036

[20] Hammer, C. (1994) Effects of endurance swimming on the growth of 0- and l-age group of whiting, Merlungius merlangus, Gadidae. Archive of Fishery and Marine Research, 42, 105-122.

[21] Trump, C.L. and Leggett, W.C. (1980) Optimum swimming speeds in fish: The problems of currents. Canadian Journal of Fisheries and Aquatic Sciences, 37, 1086-1092. http://dx.doi.org/10.1139/f80-141

[22] Fisher, R., Leis, J.M., Clark, D.L. and Wilson, S.K. (2005) Critical swimming speeds of late-stage coral reef fish larvae: Variation within species, among species and between locations. Marine Biology, 147, 1201-1212. http://dx.doi.org/10.1007/s00227-005-0001-x

[23] Brett, J.R. (1964) The respiratory metabolism and swimming performance of young sockeye salmon. Journal of the Fisheries Research Board of Canada, 21, 1183-1226. http://dx.doi.org/10.1139/f64-103

[24] Jain, K.E., Hamilton, J.C. and Farrell, A.P. (1997) Use of a ramp velocity test to measure critical swimming speed in rainbow trout (Onchorhynchus mykiss). Comparative Biochemistry and Physiology, 117A, 441-444. http://dx.doi.org/10.1016/S0300-9629(96)00234-4

[25] Bellwood, D.R. and Fisher R. (2001) Relative swimming speeds in reef fish larvae. Marine Ecology Progress Series, 211, 299-303.

[26] Mateus, C.S., Quintella, B.R. and Almeida, P.R. (2008) The critical swimming speed of Iberian barbel Barbus bocagei in relation to size and sex. Journal of Fish Biology, 73, 1783-1789.

http://dx.doi.org/10.1111/j.1095-8649.2008.02023.x

[27] Farrell, A.P. (2008) Comparisons of swimming performance in rainbow trout using constant acceleration and critical swimming speed tests. Journal of Fish Biology, 72, 693-710. http://dx.doi.org/10.1111/j.1095-8649.2007.01759.x

[28] Jones, D.R. (1971) The effect of hypoxia and anaemia on 
the swimming performance of rainbow trout (Sulmo gairdneri). The Journal of Experimental Biology Part B, 55, 541-551.

[29] Farlinger, S. and Beamish, F.W.H. (1977) Effects of time and velocity increments on the critical swimming speed of largemouth bass (Micropterus salmoides). Transactions of the American Fisheries Society, 106, 436-439. http://dx.doi.org/10.1577/1548-8659(1977)106<436:EOT AVI>2.0.CO;2

[30] Hartwell, S.I. and Otto, R.G. (1991) Critical swimming capacity of the Atlantic silverside, (Menidia menidia). Esluaries, 14, 218-221.

[31] Petersen, R.H. (1974) Influence of fenitrothion on swimming velocities of brook trout (Salvelinus fontinalis). Journal of the Fisheries Research Board of Canada, 31, 1757-1762. http://dx.doi.org/10.1139/f74-223

[32] Peake, S.J. and Farrell, A.P. (2006) Fatigue is a behavioral response in respirometer confined small mouth bass. Journal of Fish Biology, 68, 1742-1755. http://dx.doi.org/10.1111/j.0022-1112.2006.01052.x

[33] McKenzie, D.J., Claireaux, G.E. and Taylor, W. (2007) Factors influencing critical swimming speed performance in fishes. Comparative Biochemistry and Physiology, Part A1: Molecular \& Integrative Physiology, 148, S42. http://dx.doi.org/10.1016/j.cbpa.2007.06.426

[34] Yanase, K., Eayrs, S. and Arimoto, T. (2007) Influence of water temperature and fish length on the maximum swimming speed of sand flathead, Platycephalus bassensis: Implications for trawl selectivity. Fisheries Research, 84, 180-188. http://dx.doi.org/10.1016/j.fishres.2006.10.015
[35] Webb, P.W. (1975) Hydrodynamics and energetics of fish propulsion. Bulletin-Fisheries Research Board of Canada, 190, 159.

[36] Bainbridge, R. (1960) Speed and stamina in three fish. The Journal of Experimental Biology, 37, 129-153.

[37] Ryland, J.S. (1963) The swimming speeds of plaice larvae. The Journal of Experimental Biology, 40, 285-299.

[38] Zheng, Z.N. (1987) Estimation of the ocean current energy. Marine Science Bulletin, 4, 70-75.

[39] Fisher, R., Bellwood, D.R. and Job, S.D. (2000) Development of swimming abilities in reef fish larvae. Marine Ecology Progress Series, 202, 163-173. http://dx.doi.org/10.3354/meps202163

[40] Broughton, N.M. and Goldspink, G. (1978) Biochemical changes in the lateral muscles of roach, Rutilus rutilus from two habitats following exercise. Journal of Fish Biology, 13, 613-618. http://dx.doi.org/10.1111/j.1095-8649.1978.tb03474.x

[41] Jones, D.R., Kiceniuk, J.W. and Bamford, O.S. (1974) Evaluation of the swimming performance of several fish species from the Mackenzie River. Journal of the Fisheries Research Board of Canada, 31, 1641-1647.

[42] Broughton, N.M., Goldspink, G. and Jones, N.V. (1980) The effect of training on the lateral musculature of Ogroup roach, Rutius rutilus (L.), and their fatigue in subsequent exercise tests. Journal of Fish Biology, 17, 209217. http://dx.doi.org/10.1111/j.1095-8649.1980.tb02754.x

[43] Hunter, J.R. (1971) Sustained speed of jack mackerel, Trachurus symmetricus. Fishery Bulletin, 69, 267-271. 